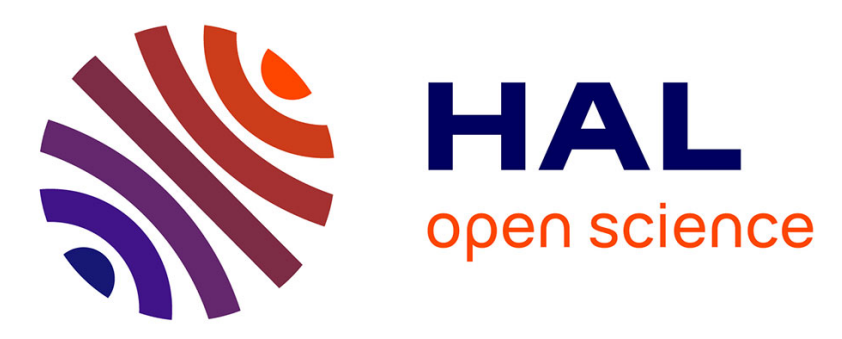

\title{
A Three-Dimensional Model of Human Lysyl Oxidase, a Cross-Linking Enzyme
}

Sylvain Vallet, Marc Guéroult, Nicolas Belloy, Manuel Dauchez, Sylvie Ricard-Blum

\section{- To cite this version:}

Sylvain Vallet, Marc Guéroult, Nicolas Belloy, Manuel Dauchez, Sylvie Ricard-Blum. A ThreeDimensional Model of Human Lysyl Oxidase, a Cross-Linking Enzyme. ACS Omega, 2019, 4 (5), pp.8495-8505. 10.1021/acsomega.9b00317 . hal-02149262

\section{HAL Id: hal-02149262 \\ https://hal.univ-reims.fr/hal-02149262}

Submitted on 6 Jun 2019

HAL is a multi-disciplinary open access archive for the deposit and dissemination of scientific research documents, whether they are published or not. The documents may come from teaching and research institutions in France or abroad, or from public or private research centers.
L'archive ouverte pluridisciplinaire HAL, est destinée au dépôt et à la diffusion de documents scientifiques de niveau recherche, publiés ou non, émanant des établissements d'enseignement et de recherche français ou étrangers, des laboratoires publics ou privés. 


\title{
A Three-Dimensional Model of Human Lysyl Oxidase, a Cross- Linking Enzyme
}

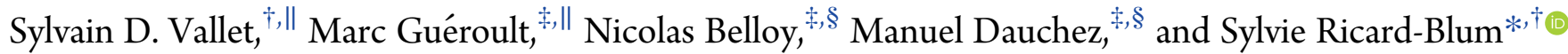 \\ ${ }^{\dagger}$ Univ Lyon, University Claude Bernard Lyon 1, CNRS, INSA Lyon, CPE, Institute of Molecular and Supramolecular Chemistry and \\ Biochemistry, UMR 5246, F-69622 Villeurbanne Cedex, France \\ ${ }^{\ddagger}$ UMR 7369 URCA/CNRS Matrice Extracellulaire et Dynamique Cellulaire (MEDyC) and ${ }^{\S}$ Plateau de Modélisation Moléculaire \\ Multi-échelle, Université de Reims Champagne-Ardenne, 51687 Reims Cedex 2, France
}

\section{Supporting Information}

ABSTRACT: Lysyl oxidase (LOX) is a cross-linking enzyme identified 50 years ago, but its $3 \mathrm{D}$ structure is still unknown. We have thus built a 3D model of human LOX by homology modeling using the X-ray structure of human lysyl oxidase-like 2 as a template. This model is the first one to recapitulate all known biochemical features of LOX, namely, the coordination of the copper ion and the formation of the lysine tyrosylquinone cofactor and the disulfide bridges. Furthermore, this model is stable during a $1 \mu \mathrm{s}$ molecular dynamics simulation. The catalytic site is located in a groove surrounded by two loops. The distance between these loops fluctuated during the simulations, which suggests that the groove forms a hinge with a variable opening, which is able to accommodate the various sizes of LOX substrates. This $3 \mathrm{D}$ model is a pre-requisite to perform docking experiments with LOX substrates and other partners to identify binding sites and to design new LOX inhibitors specific for therapeutic purpose.

\section{INTRODUCTION}

Lysyl oxidase (LOX), also known as protein-lysine 6-oxidase (EC 1.4.3.13), is a copper-dependent amine oxidase. It catalyzes the oxidative deamination of specific lysyl and hydroxylysyl residues in collagens and elastin, which is the first step of the covalent cross-linking of these extracellular matrix (ECM) proteins. ${ }^{1,2}$ Human lysyl oxidase is synthesized as a proenzyme of 396 amino acid residues, processed by bone morphogenetic protein-1 (BMP-1) and mammalian tolloids. These enzymes release the active form of LOX $(\sim 32 \mathrm{kDa})^{3}$ and its N-terminal propeptide (147 residues). The propeptide, which is extended and flexible, has been shown to interact with 34 partners, ${ }^{4}$ is required for LOX to exit the endoplasmic reticulum, ${ }^{5}$ and plays other biological roles including a ras recision gene activity, ${ }^{6}$ which categorizes it as a matricryptin. ${ }^{7,8}$ LOX contains five disulfide bridges, ${ }^{9}$ a copper ion and a lysine tyrosylquinone cofactor, ${ }^{10,11}$ which is formed autocatalytically in presence of a copper ion ${ }^{12}$ and cross-links the residues K320 and Y355 in the human enzyme. LOX shares its catalytic domain with four lysyl oxidase-like proteins (LOXL1LOXL4), but only LOXL1 contains a propeptide, which is cleaved by BMP-1. ${ }^{13}$ LOXL2-LOXL4 contain four scavenger receptor cysteine-rich (SRCR) domains at their N-terminus. Active LOX has been detected in the nuclei of fibroblasts, where it may act on histones. ${ }^{14,15}$ Other non-ECM substrates of LOX include fibroblast growth factor- $2^{16}$ and plateletderived growth factor receptor $\beta .^{17}$
LOX plays a major role in the development of the cardiovascular and respiratory systems as shown in Lox ${ }^{-/-}$ mice, which develop aortic aneurysms. ${ }^{18}$ Human LOX mutations (T248I, Q267P, S280R, M298R, and S348R, Figure 1) also predispose to aortic aneurysms and dissections in humans. ${ }^{19,20}$ Besides its role in ECM assembly and architecture, LOX is overexpressed under hypoxia and is involved in cancer progression and metastasis, particularly in breast cancer metastasis to the bone through the formation of a metastatic niche. ${ }^{21-24}$ LOX is upregulated in adipose tissue with obesity ${ }^{25}$ and in fibrosis. ${ }^{26-28}$ Targeting LOX in fibrosis and cancer may thus be a new therapeutic approach in these diseases. ${ }^{23,29-31} \beta$-Aminopropionitrile is a potent inhibitor of LOX activity, but its toxicity limits its use as a drug.

The design of new inhibitors of LOX catalytic activity and/ or of specific interaction of LOX with one of its partners requires the knowledge of the 3D structure of LOX, which has not been determined so far, although the enzyme has been discovered 50 years ago. ${ }^{32}$ However, LOX becomes highly insoluble upon enzymatic release from the prolysyl oxidase and is thus very difficult to express in a soluble form and in sufficient amount for structural studies using X-ray crystallography and/or NMR spectroscopy. ${ }^{6}$ Two high-resolution crystal

Received: February 3, 2019

Accepted: May 3, 2019

Published: May 14, 2019 
169 DDPYNPYKYS DDNPYYNYYD TYERPRPGGR YRPGYGTGYF QYGLPDLVAD 219 PYYIQASTYV QKMSMYNLRC AAEENCLAST AYRADVRDYD HRVLLRFPQR 269 VKNQGTSDEL PSRPRYSWEW HSCHQHYHSM $\stackrel{*}{*}$ DEFSHYDLID ANTQRRVAEG 319 HKASFCLEDT SCDYGYHRRF ACTAHTQGLS PGCYDTYGAD IDCEWIDITD 369 VKPGNYILKV SVNPSYLVPE SDYTNNVVRC DIRYTGHHAY ASGCTISPY

Figure 1. Prediction of the secondary structure (PROTEUS) and intrinsic disorder (metaPrDOS) in the sequence of human lysyl oxidase. Residues predicted to be intrinsically disordered are underlined, residues predicted to form $\alpha$-helices are in red, and those forming $\beta$-strands are in green. Cysteine residues involved in disulfide bonds are connected by bold black links. K320 and Y355 residues forming the lysine tyrosylquinone cofactor are connected by a bold blue link. Histidine residues coordinating the copper ion are boxed in yellow. Asterisks (*) denote to mutations identified in patients. ${ }^{19,20}$

structures of Pichia pastoris lysyl oxidase (PPLO) are available in the Protein Data Bank (PDB, http://www.rcsb.org; PDB IDs: $1 \mathrm{~N}^{3} \mathrm{E}^{33}$ and $1 \mathrm{~W} 7 \mathrm{C}^{34}$ ), but PPLO is dimeric and contains a topaquinone cofactor (TPQ), whereas human LOX is monomeric and has a lysine tyrosylquinone cofactor. PPLO is thus not a good enough template for the homology modeling of human LOX. Two in silico models of human LOX have been published, but they do not include and/or do not fit to all the experimental molecular and structural data available for LOX $^{35,36}$ A third model encompasses only a short sequence of human LOX (residues Y284-F301) and focuses on the coppercoordination site. ${ }^{37}$ The publication of the X-ray structure of human LOXL2 $2^{38}$ prompted us to use it as a template to generate a $3 \mathrm{D}$ model of human LOX including the copperbinding site, the LTQ cofactor, and the five disulfide bridges. Indeed, the sequences of human LOX and LOXL2 catalytic domains share $49 \%$ of sequence identity. The resulting model fits well to all biochemical and structural information available. The stability of the model was then assessed by a long molecular dynamics simulation $(1 \mu \mathrm{s})$.

\section{RESULTS}

Sequence Analysis of Human Mature Lysyl Oxidase. The percentages of $\alpha$-helices and $\beta$-strands predicted in the sequence of human LOX were 17 and $18 \%$, respectively, and the percentage of random coil was $65 \%$ (Figure 1). A sequence of 29 residues enriched in tyrosine residues and located at the $\mathrm{N}$-terminus and a short sequence of three residues at the $\mathrm{C}$ terminus were predicted to be intrinsically disordered (Figure $1)$.

Prediction of Molecular Recognition Features and Protein-Binding Regions. According to MoRFchibi SYSTEM, the D169-P195 and R198-Y203 sequences of LOX would be able to undergo a disorder-to-order transition upon binding to its partners. The intrinsically disordered sequence ${ }^{181}$ NPYYNYYD ${ }^{188}$ located at the N-terminus of LOX was also predicted by ANCHOR to be able to fold upon binding. This sequence is present in bovine, chicken, human, murine, and rat LOX and was also found in unreviewed UniProtKB entries of LOX using BLAST but not in other proteins. It thus appears to be specific of LOX.

Homology Modeling of Lysyl Oxidase. The first 3D structure of a member of the LOX family, human LOXL2, has been recently published. ${ }^{38}$ The sequence of the catalytic domain of human LOXL2 (P548-S751) shares 49\% of identity with that of human LOX (P213-Y417), which allowed us to build a 3D model of LOX by homology modeling. Ten thousand models were generated with the comparative modeling protocol of Rosetta ${ }^{39}$ including the copper ion and the lysine tyrosylquinone cofactor. The coordination site of the copper ion was restrained by harmonic potential between the $\mathrm{C} \beta$ of histidine residues involved in copper ion coordination. A harmonic potential was also applied between the nitrogen atom of K320 and the oxygen atom of Y355. The flat bottom of these potentials was defined by the distance observed in the X-ray structures of LOXL2 (PDB ID: 5ZE3) and of lysyl oxidase from $P$. pastoris (PDB IDs: 1N9E and 1W7C).

Both LOX and LOXL2 contain 10 conserved cysteine residues in their catalytic domain, namely, C238-C244, C291C340, C324-C330, C351-C361, and C398-C412 for LOX, ${ }^{9}$ and C663-C685, C673-C657, C579-C695, C625-C573, and C732-C746 for LOXL2, ${ }^{38}$ but they are paired differently except for one of them (Figure S1).

Four out of the 5 disulfide bonds of LOX were formed during the first step of modeling using Rosetta. The C291C340 disulfide bridge was not formed at this stage indeed. The sequence encompassing the catalytic domain of LOX (residues P213-Y417) shared a common architecture with the catalytic domain of LOXL2 (residues P548-S751; maximum RMSD C $\alpha: 1.75 \AA$ ) (Figure 2A). The C291-C340 disulfide bridge

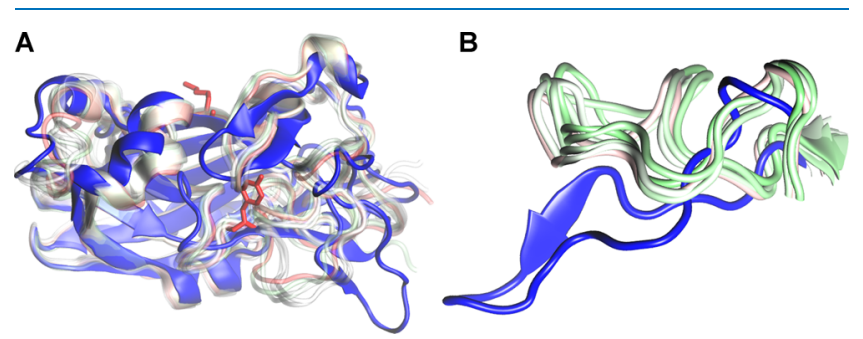

Figure 2. Superimposition of the five best models generated for the catalytic domain of LOX (P213-Y417) selected with ProQ2 and of the X-ray structure of LOXL2 (PDB ID: 5ZE3; P548-S751, in blue). (A) Global view of the catalytic domain and (B) detailed view of loop 10 (D331-G350 in LOX). The residues involved in LTQ formation in the X-ray structure of LOXL2 are colored red. They are too far from each other and are not in the correct orientation to form the LTQ in the crystal structure of LOXL2.

could lock/constrain the bottom of the D331-G350 loop of LOX, which corresponds to loop 10 in LOXL2 (Figure 2B). These conserved cysteine residues are not paired together in the X-ray structure of LOXL2.

These best models were then refined with Rosetta to form the missing disulfide bridge between C291 and C340. When the five disulfide bridges of LOX were formed, both the K320 and Y355 were close enough to form the LTQ cofactor without further external constraints, which is observed for the first time in a model of this protein. Moreover, these amino acids were close enough to the $\mathrm{Cu}^{2+}$ coordinated by the three histidine residues previously identified in LOX (H292, H294, and H296). ${ }^{26}$ The LOX model (Figure 3) comprised $9.2 \%$ of helices, $18.9 \%$ of $\beta$-strands, and $71.9 \%$ of random coil and turns, as determined with DSSP (Figure $3 \mathrm{~A}$ and Table S1). The percentage of random coil in the model was similar to that predicted in the sequence of human lysyl oxidase by 

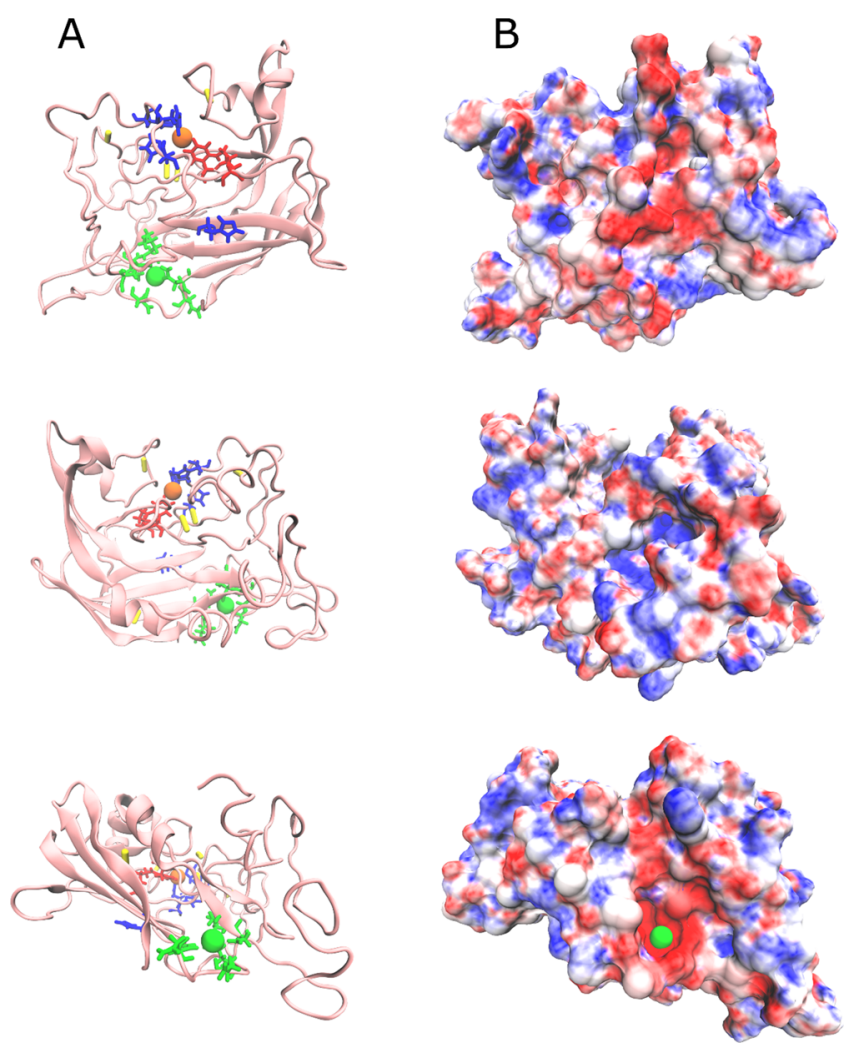

Figure 3. (A) Refined 3D model of human LOX (residues D169Y417) in three orientations. Orange: copper; green: calcium; red: LTQ; yellow: disulfide bonds; blue: histidine residues coordinating the copper ion. The calcium coordination site identified in LOXL2 (D549, L550, E722, D724, N727, and N728) is conserved in LOX (D214, L215, E388, D390, N393, and N394; residues in green). (B) Electrostatic potential of the LOX model rendered on the molecular surface $( \pm 10 \mathrm{kT} / \mathrm{e}$, acidic residues in red, basic residues in blue), without contribution of ions, was generated using the PDB2PQR server $^{42}$ (http://nbcr-222.ucsd.edu/pdb2pqr_2.0.0/).

PROTEUS (65\%), which does not discriminate random coil and turns.

The N-terminal sequence of LOX encompassing the residues 169-212 differs from that of LOXL2, and the absence of a template prevented the homology modeling of the $\mathrm{N}$-terminus of LOX. A sequence homologous to the cytokine receptor class 1 has been identified in the LOX sequence (residues C351-C412). ${ }^{40}$ The structural alignment of the 3D structures of this domain from human erythropoietin receptor
(PDB ID: $1 \mathrm{ERN}^{41}$ ) with our 3D model of LOX presents a correct overall shape of the domain with a correct superposition of the $\beta$-sheet in the interface with the other domain of the model (Figure S2).

Coordination of Copper and Calcium lons in the LOX Model. LOX and LOXLs are both copper-dependent enzymes. Restraints were thus applied on $\mathrm{Cu}^{2+}$ in the LOX model to keep it coordinated by histidine residues $\left(\mathrm{N}^{\varepsilon}\right.$ for $\mathrm{H} 292$ and $\mathrm{H} 294$ residues and $\mathrm{N}^{\delta}$ for $\mathrm{H} 296$ residue) and close from the LTQ. They were based on data from the copper amine oxidase family ${ }^{33,34}$ and on the corresponding coordination site in the X-ray structure of LOXL2, where a zinc ion replaces the copper ion. Furthermore, as indicated above, a calcium ion, observed in the catalytic site of LOXL2, was added in the equivalent site of the LOX model. The H292, $\mathrm{H} 294$, and $\mathrm{H} 296$ residues coordinating the copper ion and the LTQ cofactor were obtained in a coherent structural environment with the copper ion and the oxygen atom of the quinone (Figure 4A). No positional restraint was applied to the histidine residue $\mathrm{H} 303$, which might be involved in the catalytic mechanism of the enzyme, ${ }^{43}$ because the corresponding histidine residue in LOXL2 (H637) is not in the first coordination shell of the zinc ion $(\mathrm{dZn}-\mathrm{H} 637=14.8 \AA$ ). The simulations revealed that this histidine residue had no structural influence. There were no well-defined cationic patches on the surface of LOX model. However, two anionic clusters were observed, one of them containing the residues coordinating the calcium ion (Figure 3B). The calcium ion was accessible on the model surface of the model (Figure 4B), in contrast to the copper ion, which is buried inside the model (Figure 3B). This is consistent with the experimental data indicating that the copper ion is tightly bound to the protein. ${ }^{44}$

Molecular Dynamics Simulations. To evaluate the dynamics and stability of the 3D model of LOX, molecular dynamics simulations were performed for a total trajectory of 1 $\mu$ s. A deviation from the initial LOX model was observed during the first $300 \mathrm{~ns}$ of the simulation (RMSD C $\alpha$ : $5.02 \pm$ $0.41 \AA$, Figure 5A). The largest deviation from the initial model was observed between residues D169 and P213, corresponding to the LOX sequence for which no template was available in LOXL2 (data not shown). The distance between the $\mathrm{C} \alpha$ of $\mathrm{D} 169$, located at the N-terminus of LOX, and the geometric center of the $\mathrm{C} \alpha$ of the amino acid residues of the LOX sequence (P213-Y417) varied significantly (20.4 \pm $4.6 \AA)$ during the first $300 \mathrm{~ns}$ of the simulation, but the model was more stable afterward $(9.5 \pm 0.8 \AA$ ) (Figure $5 \mathrm{~B})$.

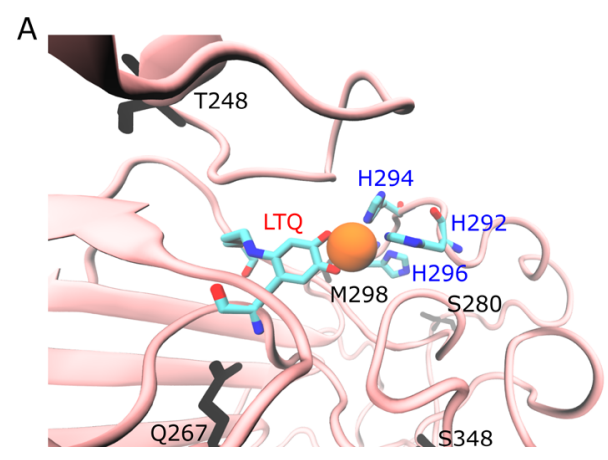

B

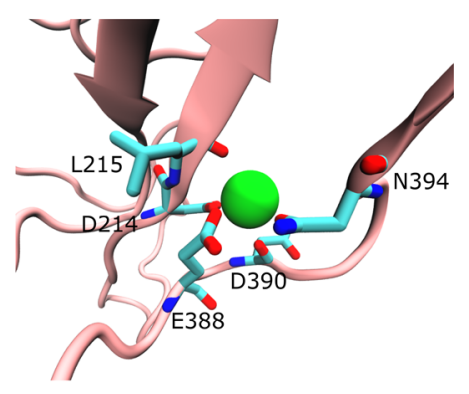

Figure 4. (A) Part of the human lysyl oxidase model encompassing the three histidine residues coordinating the copper ion and the LTQ cofactor and (B) coordination site of the calcium ion within the LOX model. 

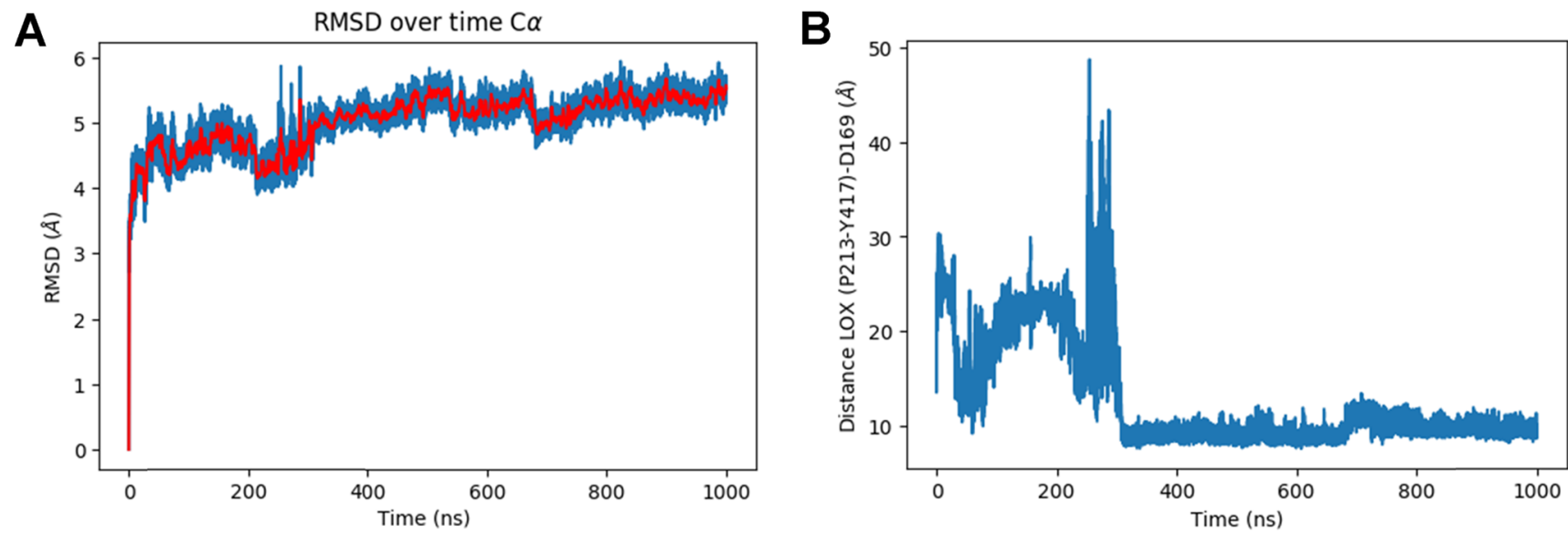

Figure 5. (A) Variation of the RMSD measured on the $\mathrm{C} \alpha$ during the simulation. (B) Distance between the C $\alpha$ of D169, located at the N-terminus of LOX, and the geometric center of the $\mathrm{C} \alpha$ of the amino acid residues of the sequence of LOX (P213-Y417) during the simulation.

The RMSD was smaller $(\mathrm{C} \alpha=1.98 \pm 0.54 \AA)$ during the last $600 \mathrm{~ns}$ of the simulation using the $400 \mathrm{~ns}$ snapshot as a reference (Figure S3). In addition, two structural states of the model were observed during this part of the simulation. These two states were also observed when plotting the distribution of the distance between the S232-H259 and Q272-F301 loops, located at the entrance of LOX catalytic site, as a function of the $\mathrm{C} \alpha$-RMSD over the last $600 \mathrm{~ns}$ of the simulation (Figure S4).

The proportion of secondary structures, calculated with DSSP, did not vary significantly during the simulation (Figure S5). The sequences forming $\beta$-strands remained very stable over the stimulation. The major fluctuations took place in the $\mathrm{N}$-terminal sequence, which forms either $\alpha$-helices or turns in the course of the simulation, consistent with the fact that the $\mathrm{N}$-terminus of LOX is enriched in intrinsic disorder as predicted by metaPrDOS and is thus flexible. The two major coiled regions (189-199 and 319-329 residues) did not form any secondary structure during the simulation. The lack of formation of the secondary structure in the sequence 319-329 during the simulation is consistent with the disulfide bridge connecting the cysteine residue 324 with the cysteine residue 330 and the presence of the lysine residue 320 involved in the formation of the LTQ which both restrain the motion of this sequence. However, the average content of helices (12\%), $\beta$ strands $(23 \%)$, and random coil and turns $(65 \%)$ was higher than those calculated before the simulation.

The general dynamics of the 3D LOX model was compared to the X-ray temperature factor of the LOXL2 structure (Figure 6). The fluctuations were evaluated during the last 700 ns of the simulations, where the LOX model was the most stable. The data generated for LOX and LOXL2 were very similar. The fluctuations observed during the MD simulations of the LOX model followed the same trend than those of the B-factor profile for residues 230-300 and 325-360 (Figure 6). The difference in cysteine pairing between LOX and LOXL2 could contribute to the differences in fluctuations.

Molecular dynamics was performed in the presence of one copper ion, one calcium ion, and five potassium ions. All the residues involved in calcium and potassium coordination are conserved in LOX through evolution (250 sequences retrieved from UniRef90 ${ }^{45}$ ) (Figure S6). The coordination sphere and the amino acid residues coordinating the ions were evaluated with a radial distribution function. ${ }^{46}$ The copper coordination

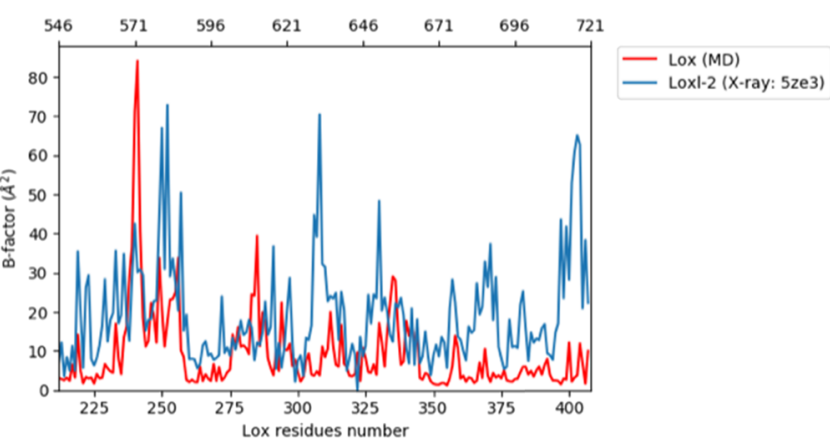

Figure 6. Comparison of the B-factor profile of the X-ray structure of LOXL2 (residues T546-A721, blue) with the fluctuations of the LOX model (residues L212-Y408, red) during the trajectory.

sphere was defined by 2.45 and $4.4 \AA$ distances (Figure 7A), in agreement with expected values, and adopted an octahedral geometry (Figure S9). The coordination number associated with the first coordination shell was 5.9 (Table S4). The coordination sphere of the copper ion was defined by the LTQ, H292, H294, and H296 residues. Although all these residues were constrained to coordinate the copper ion (Figure 7B), the H296 residue did not interact directly with the copper ion but stabilized a water molecule involved in the copper coordination sphere (Figure S9).

The calcium coordination distance was $2.60 \AA$ A (Figure 7A), the coordination number was 8 (Table S4), and the coordination sphere had an atypical square antiprismatic molecular geometry (Figure S9), which has been described in a bacterial collagen-binding domain. ${ }^{47}$ The calcium ion remained located within the coordination site constituted by the residues D214, L215, E388, D390, and N394 during the simulation. Its residence time was evaluated during the entire simulation and was found to be $99.96 \%$ for D214, $99.81 \%$ for L215, 100\% for E388, 99.87\% for D390, and $58.41 \%$ for N394 (Figure 7C).

The coordination distance was $2.74 \AA$ for the potassium ion, with a small contribution of the second sphere at $4.5 \AA$ (Figure $7 \mathrm{~A})$. During the last $130 \mathrm{~ns}$ of production, the potassium ion bound to LOX via L325 (82.86\%), D327 (94.00\%), H343 (61.19\%), G346 (80.86\%), and S348 (55.96\%) residues (Figure 7D). The potassium ion interacted with L325, G346, and S348 via the backbone and with D327 and H343 via their side chains (Figure S9). The coordination sphere of the 

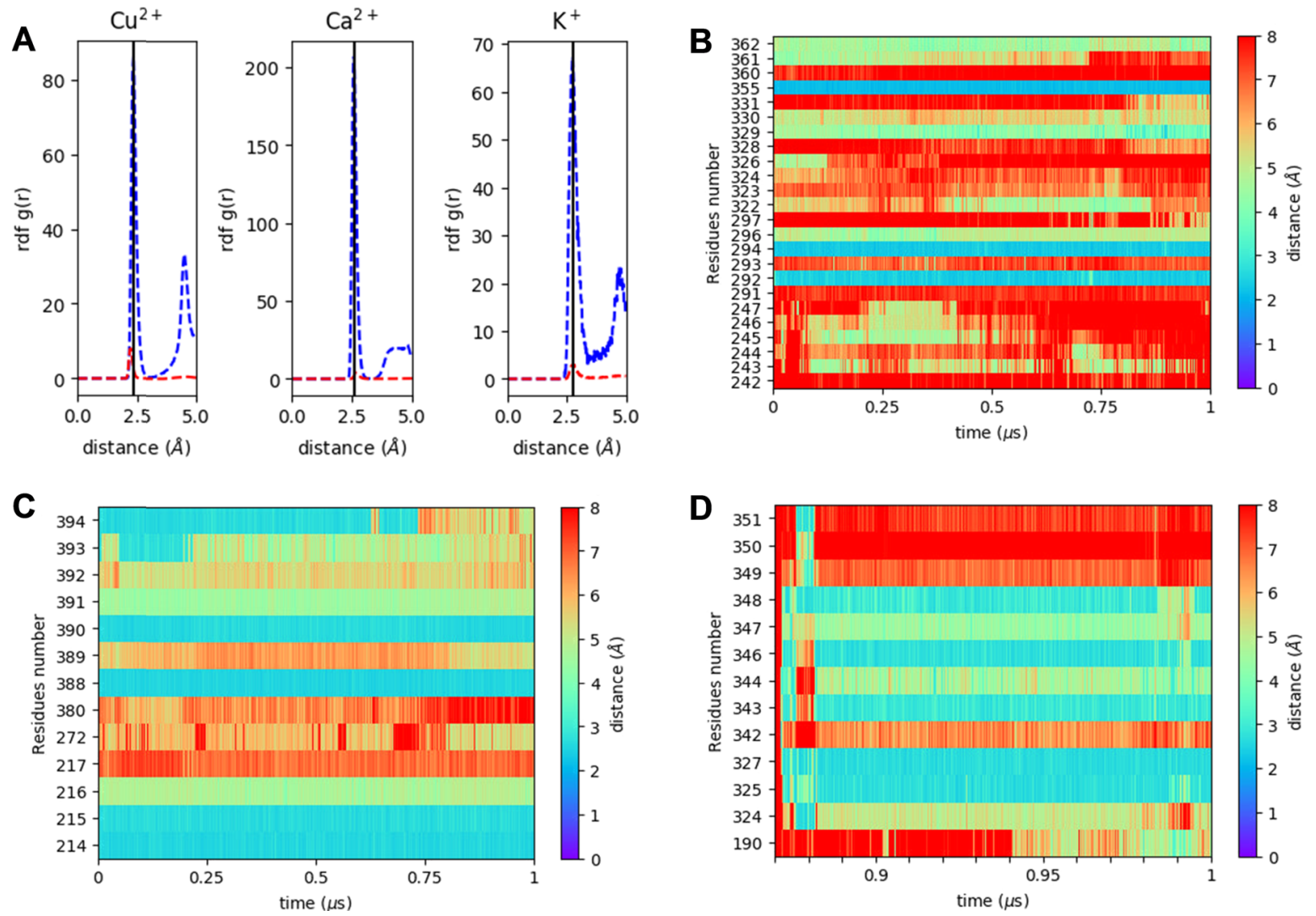

Figure 7. (A) Coordination sphere of the copper, calcium, and potassium ions evaluated with a radial distribution function (rdf). Amino acid residues located within a $5 \AA$ of cations (B) copper ion, (C) calcium ion, and (D) potassium ion. The matrix distance was calculated for the whole simulation for the copper and calcium ions ( $\mathrm{B}$ and $\mathrm{C}$ ) and was focused on the last $130 \mathrm{~ns}$ of the MD simulation for the potassium ion.

A

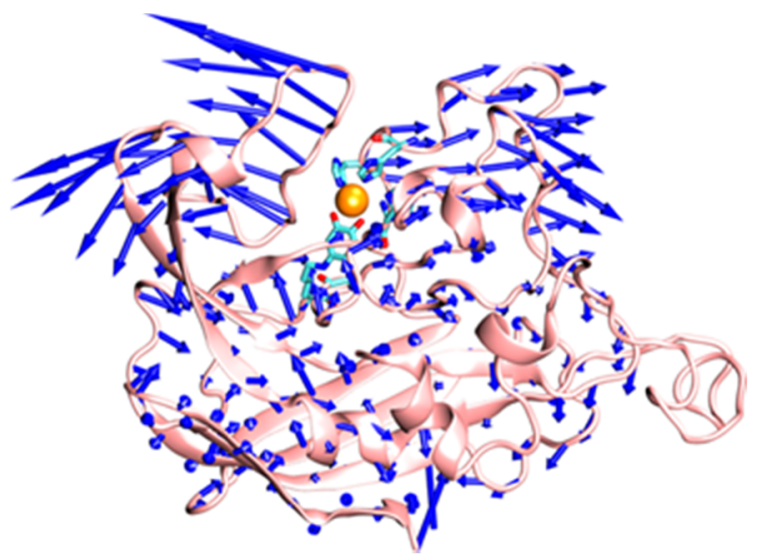

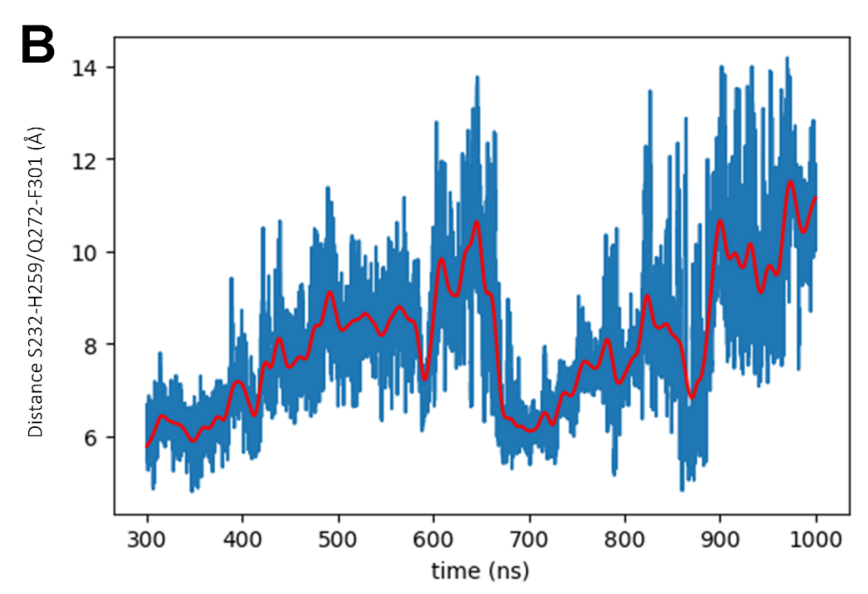

Figure 8. (A) Essential dynamics: Schematic representation of the displacement of $\mathrm{C} \alpha$ (blue arrows indicating the direction and the amplitude of the motions; orange: copper ion; $\mathrm{His}^{292}, \mathrm{His}^{294}, \mathrm{His}^{296}$, and the LTQ cofactor are represented). (B) Distance between the loops S232-H259 and Q272-F301 measured during the simulation (smoothed curve: red).

potassium ion adopted a dodecahedral geometry (Figure S9), and the coordination number of potassium ion was 7.5 (Table S4). The number of coordination is high for a potassium ion since the usual coordination number is 6 . However, this site identified in the last $130 \mathrm{~ns}$ of the simulation could be occupied in vivo by another cation (e.g., a divalent cation such as calcium).

The catalytic site is located in a groove surrounded by two loops, S232-H259 and Q272-F301, leading to a V shape of the catalytic site. The distance between the above loops was measured during MD simulations to follow the accessibility of the catalytic site. The distance between them fluctuated from 4.8 to $14.2 \AA$, suggesting that the two structural states identified during the last $600 \mathrm{~ns}$ of the simulation correspond to an open state and a closed state. To better characterize the groove opening, essential dynamics was performed on the last $600 \mathrm{~ns}$ of the simulation over the backbone $(\mathrm{C} \alpha)$ of the catalytic domain based on the C $\alpha$-RMSD to extract the correlated motions of LOX and to determine the motions that are important for its activity. The analysis of the first 25 principal components (PC) showed that the first six eigenvectors accounted for $80 \%$ of the global motion of 

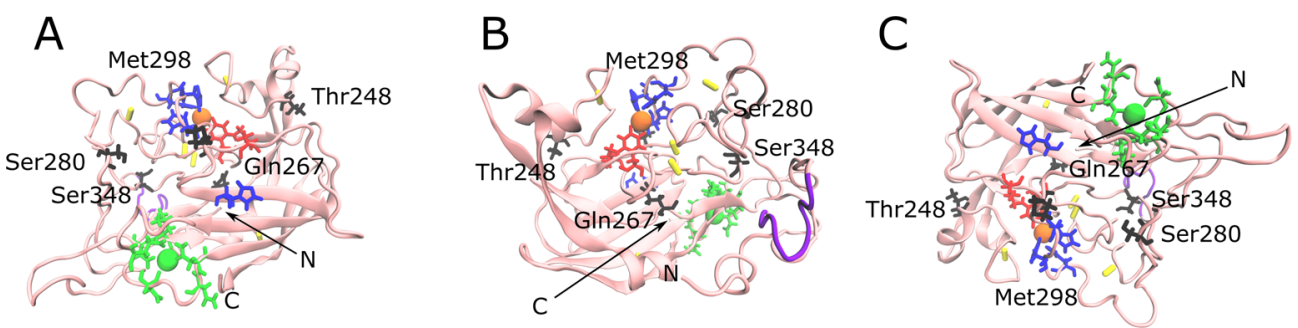

Figure 9. (A-C) Refined 3D model of human LOX (residues P169-Y417) in three orientations. Copper: orange; LTQ: red; yellow: S-S bonds; calcium: green. The calcium coordination site identified in LOXL2 (D549, L550, E722, D724, N727, and N728) is conserved in LOX (D214, L215, E388, D390, N393, and N394; residues in green). Histidine residues coordinating the copper ion: blue. Unique LOX sequence (NPYYNYYD; N181-D188 is in purple). Mutations (black) inhibiting LOX enzymatic activity (percentage of inhibition in brackets) and associated with aortic aneurysms and dissections in humans, ${ }^{19,20}$ namely, T248I (8\%), Q267P (not determined), S280R (50\%), M298R (100\%), and S348R (21\%). N: N-terminus; C: C-terminus.

LOX (Figure S7). To characterize the motion between the open and closed states using these eigenvectors, the corresponding structural motion was further investigated. The contribution of each of the six eigenvectors to the residue fluctuations showed that both PC1 and PC3 are responsible for the opening motion. The PC3 characterized the large motion of the loop S232-H259 (Figure 8). This suggests that the groove forms a hinge with a variable opening, which could accommodate various sizes of substrates in agreement with the known enzymatic activity of LOX on small (e.g., FGF2) and large (e.g., collagens) proteins. The maximum opening of the groove (Figure 8) was consistent with the diameter of a collagen molecule $(\sim 15 \AA$ including side chains; PDB ID: $2 \mathrm{KLW})$. When considering the motions observed in the trajectories, the $\mathrm{H} 303$ residue, which might act as a base during the catalytic process, ${ }^{43}$ appeared to be positioned at the bottom of the groove and might be activated whatever the groove opening.

\section{DISCUSSION}

This model is the first one to recapitulate all known molecular features of human mature LOX, namely, copper coordination residues, the LTQ cofactor, and the five disulfide bridges. Indeed, disulfide bridges were not included in both in silico models previously published. Kagan and Ryvkin's model ${ }^{35}$ was built with Schrödinger, Inc. Molecular Modeling Software using sequence alignments and secondary structure prediction, and contained the copper ion and the LTQ. However, in this model the LTQ is located at $20 \AA$ from the copper ion, which is at odds with its redox role during the catalytic process. Furthermore, Kagan and Ryvkin did not perform molecular dynamics simulations to assess the structural stability of their model. The second published model ${ }^{36}$ was built ab initio with the Robetta server and refined with MAESTRO 9.3 but does not contain the LTQ cofactor. ${ }^{36}$ A very short dynamics simulation (4 ns) was performed, but it was not sufficient to evaluate the stability of the proposed model, and the fluctuations during the trajectory were not reported.

Furthermore, it is difficult to compare the content in secondary structures and random coils of our model with Kagan and Ryvkin's model because the total amount of secondary structures of their model was higher than $100 \%$ (110\%: $20 \% \alpha$-helices, $25 \% \beta$-strands, $65 \%$ random coil, and turns). On the other hand, our model contains less secondary structures than Bhuvanasundar's model ${ }^{36}$ (24 and 25\% of $\alpha$ helices and $\beta$-strands, respectively, for their model versus 9.2 and $18.9 \%$ of $\alpha$-helices and $\beta$-strands, respectively, for our model). The values calculated from the deconvolution of circular dichroism spectra of human LOX are twice higher than in our model for $\alpha$-helices (21\%) and in the same range for $\beta$ strands $^{26}(27.5 \%)$. Our 3D model contains approximately $69 \%$ of random coil and turns, in agreement with the amount of disorder and turns predicted by PROTEUS (65\%), but it is higher than the amount of random coil calculated by circular dichroism (52\%). ${ }^{26}$ The N-terminal sequence NPYYNYYD, predicted as intrinsically disordered, is indeed disordered in the 3D model. This sequence is only found in LOX and not in LOX-like proteins and might be involved in specific interactions of LOX with unique substrate and/or nonsubstrate proteins.

A calcium binding site, identified in human LOXL2, is conserved in human LOX, and it is located at a distance from the catalytic site. Several LOX binding partners and substrates are calcium-binding proteins (e.g., elastin, fibulin-5, bone morphogenetic protein-1, tolloid-like protein 1, perlecan, and fibrillin-1) or play a role in calcium homeostasis (stanniocalcin 2 ). The calcium-binding site of LOX could tether these substrates to the LOX surface and position them in an appropriate orientation to be oxidatively deaminated within the catalytic site. The effect of calcium on LOX enzymatic and/or biological activities has to be investigated. The calcium ion remained stable during the $1 \mu \mathrm{s} \mathrm{MD}$ trajectory and might contribute locally to the stability of the model. It should be noted that loop 10 became closer to the calcium coordination site after $600 \mathrm{~ns}$ of simulation, but its position fluctuates during the last part of the simulation.

The T248I, S280R, and S348R mutants, which predispose to aortic aneurysms and dissections in humans ${ }^{19,20}$ and are the most distant from the catalytic site, inhibit the activity of LOX to a lesser extent than the M298 mutant, ${ }^{19,20}$ which is closer to the catalytic site and abolishes LOX activity (Figure 9). However, the mutation of the S348 residue, which is located at the bottom of the groove, decreases the activity by $50 \%,{ }^{19}$ emphasizing the potential role of the groove in the catalytic mechanism. In the vicinity of this residue, a potassium ion is observed in the first sphere of coordination (2.74 $\AA$, during $56 \%$ of the last $130 \mathrm{~ns}$ of the trajectory). The serine-to-arginine mutation, replacing a polar amino acid by a longer, positively charged residue, could lead to the loss of potassium or any other cation and may be important for the activity of the LOX.

We used MAESTRO, a Multi Agent Stability Prediction tool, for changes in unfolding free energy upon point mutation (https: / / biwww.che.sbg.ac.at/maestro/web) ${ }^{48,49}$ to predict the stability change expressed as $\Delta \Delta G$ induced by the mutations. 
MAESTRO accepts 3D models as an input. All the mutations, T248I, Q267P, M298R and S348R, destabilize the protein. M298R, which lacks significant enzyme activity, has the strongest destabilizing effect (Table S2), whereas the destabilizing effects of T248I and S348R, which decreases LOX activity by 8 and $21 \%,{ }^{19}$ are less pronounced. The only discrepancy in mutation analysis concerns the S280R mutation, which is predicted to stabilize the protein and is located at a higher distance from the copper ion and LTQ than the other mutated amino acid residues (Tables S2 and S3), although it decreases the enzyme activity by $50 \%$. However, no role has been reported for this serine residue in the catalytic mechanism.

As mentioned above, potassium ions were added in the simulation to perform simulations in neutral condition. After $750 \mathrm{~ns}$, one of the potassium ions was stabilized in a cleft of coiled parts of the enzyme and remained here until the end of the trajectory. This potassium ion seems to play a key role in the local stabilization of this flexible part of the $3 \mathrm{D}$ structure.

The model of the human LOX reported here is the first one to include the five disulfide bridges stabilizing its tertiary structure, as well as the copper ion and the LTQ cofactor, both of which are required for its enzymatic activity, ${ }^{26}$ whereas the only crystal structure available for a member of the mammalian LOX family does not contain the LTQ cofactor and copper ion and represents a zinc-bound precursor state requiring conformational changes for its activation. ${ }^{38}$ Numerical simulations taking into account all these molecular characteristics were performed in a solvated environment, leading to a long trajectory of $1 \mu \mathrm{s}$. The stability of the overall 3D structure was conserved along the trajectory. The secondary structure elements were also stable during the simulations. The content in secondary structures may require optimization, but it does not significantly influence the global fold of our model. The nonstructured parts provide LOX with the structural flexibility and dynamics required for its adaptation to substrates of various sizes and shapes, from growth factors to collagen fibrils. The LTQ remains in a correct position during the simulation, acting as a "bottom floor" in the inner core of the protein, and is crucial for the coordination of the copper ion. The copper ion and the three histidine residues are also correctly positioned during the whole simulations. The solvation of the groove, where there are numerous water molecules, will be of interest for structural studies of complexes formed by LOX with its natural substrates.

The disulfide pattern of the LOX model differs from the pattern observed in the X-ray structure of LOXL2. Only one of the five disulfide bonds was conserved. We generated an alternative model of LOX with Swiss-Model ${ }^{50}$ using the X-ray structure of LOXL2 as a template to determine the influence of the cysteine pairing pattern on the folding of LOX. The fold of this homology model containing the disulfide pattern of LOXL2 was similar to the fold of the dynamic model of LOX $(\mathrm{RMSD}=3.4 \AA$, Figure S8). Both models had common secondary structures, although the homology model contained few extra helix-forming residues and three to four further short $\beta$-strands (Figure S8 and Table S1). The residues involved in the LTQ formation are not close enough and not well oriented in the homology model to allow the formation of the cofactor. Although the pattern of LOXL2 disulfide bonds is plausible for LOX, it does not allow the formation of the LTQ cofactor, whereas the pattern of LOX disulfide bonds allows the formation of LTQ. This is in agreement with the fact that high- similarity proteins do not necessarily share the same connectivity patterns of cysteine residues, as shown for scorpion toxins. ${ }^{51}$ Scorpion toxins MTX and Pi4 have a high sequence identity $(81.6 \%)$ and the same number of cysteine residues but different connectivity patterns, whereas scorpion toxins BMTX1 and LpII have a low sequence identity (21\%) but share an identical cysteine pairing pattern. ${ }^{51}$ It should be noted that the X-ray structure of LOXL2 represents a zincbound precursor state according to the authors. Pronounced conformational rearrangements would be required for protein activation, ${ }^{38}$ and this may include the reshuffling of disulfide bonds. It is thus likely that our model of LOX and the crystal structure of LOXL2 represent different states, namely, a potentially active state of LOX for our model and a nonfunctional, inactive precursor state for the X-ray structure of LOXL2.

\section{CONCLUSIONS}

We have built the first 3D model of human LOX encompassing all the major features required for its enzymatic activity (the LTQ cofactor and coordination site of the copper ion) and the five disulfide bridges stabilizing its 3D structure. This enzyme, known for several decades, is implicated in numerous biochemical and physiopathological processes, but its 3D structure was never solved at the atomic level by crystallography or NMR. The model proposed in this work takes into account all the different structural features in the literature and is consistent with the few experimental biophysical and structural information reported in the literature. If some of the secondary structures have to be refined in the model, the coil parts of the protein seem to be important to allow the plasticity and, consequently, the function of the enzyme. The long simulations performed have proved the stability of the built model, and the dynamical features are consistent with the known functions of LOX. This model will be useful to perform docking experiments with substrates and other binding partners to decipher the molecular mechanisms governing these interactions. This model will be also useful for docking small molecules potentially acting as LOX inhibitors. It will be useful to design new inhibitors specific of LOX for therapeutic purpose.

\section{MATERIAL AND METHODS}

Prediction of Secondary Structure and Intrinsic Disorder of Human LOX. The secondary structure of human LOX (UniProtKB accession number P28300) was predicted with PROTEUS Structure Prediction Server $2.0^{52}$ (http://www.proteus2.ca/proteus/index.jsp).

Intrinsic disordered regions were predicted using metaPrDOS, combining DISOPRED2, DISPROT, IUPred, and PrDOS predictors with a ROC score of $0.897 .{ }^{53}$ Disordered regions of LOX that were able to undergo disorder-to-order transition upon binding to its partners were predicted with ANCHOR $^{54,55}$ (https://iupred2a.elte.hu/), and Molecular Recognition Features (MoRFs) were predicted using MoRFchibi SYSTEM ${ }^{56}$ (https://gsponerlab.msl.ubc.ca/software/ morf_chibi/).

The Multi Agent Stability Prediction (MAESTRO, https:// biwww.che.sbg.ac.at/maestro/web/ $)^{48,49}$ tool for changes in unfolding free energy upon point mutation was used to evaluate the effect of mutations found in patients on the 
stability of lysyl oxidase (MAESTRO provides $\Delta \Delta G$ values with prediction quality measures.

Homology Modeling of Human Lysyl Oxidase. Several approaches including data reported in the literature were used to build a 3D model of human LOX. To generate the LOX model, we used Rosetta software, allowing us to combine both threading and comparative modeling approaches through the RosettaCM protocol. ${ }^{39}$ For the threading approach, fragment libraries of three and nine amino residues (371 and 2373 fragments, respectively) were built using the Robetta server (http://robetta.bakerlab.org/). ${ }^{57}$ The best templates for comparative approach were identified using the Basic Local Alignment Search Tool (BLAST) against the PDB, with the LOXL2 X-ray structure having been recently solved (PDB ID: $\left.5 \mathrm{ZE} 3^{38}\right)$.

HHpred $^{58,59}$ (https://toolkit.tuebingen.mpg.de/\#/tools/ hhpred) was used to retrieve distant homologs of the human lysyl oxidase sequence (residues 169-417) based on hidden Markov models (HMMs). Rosetta ${ }^{39}$ was then used with the RosettaCM procedure (Comparative Modeling) to generate 10,000 models based on the Robetta fragment libraries, the catalytic domain of human LOXL2 ${ }^{38}$ (PDB ID: 5ZE3) (residues 548-751 corresponding to the LOXL domain annotated in UniProtKB), and the structural data available, namely, a copper ion coordinated by H292, H294, and H296 residues, the cofactor LTQ involving $\mathrm{K} 320$ and $\mathrm{Y} 355$, and five disulfide bonds (C238-C244, C291-C340, C324-C330, C351C361, and C398-C412). ${ }^{9}$

The distance between the atoms of the residues forming the LTQ namely, the nitrogen atom of K320 side chain and the oxygen atom of $\mathrm{Y} 355$ side chain, was constrained by a harmonic potential to keep it closer to $5 \AA$. The LOX model was fitted to the catalytic site of the LOXL2 template on the $\mathrm{C} \beta$ of the three histidine residues coordinating the copper ion and on the $\mathrm{C} \alpha$ of the lysine and tyrosine residues forming the LTQ cofactor issued from the X-ray structure of the D298K mutant of the copper amine oxidase from Arthrobacter globiformis (PDB ID: $2 \mathrm{YX}^{60}$ ). This enzyme was used as a template to generate the LTQ because the copper-binding site of LOXL2 is occupied by zinc in the X-ray structure, which prevents the formation of a LTQ. Furthermore, the copper amine oxidase from $A$. globiformis is the only structure available in the PDB, which contains a LTQ cofactor. The D331-G350 sequence of LOX, aligned with the loop 10 of LOXL2, was refined with Rosetta script on the 10 best templates evaluated with ProQ2 assessment program ${ }^{61}$ to allow the formation of the C291-C340 disulfide bridge, which was not formed during the first step of modeling, although it was given as a constraint to Rosetta. The atomic coordinates of the final model of human lysyl oxidase is provided in the Supporting Information.

Molecular Dynamics Simulations. Starting from this model, the structure was minimized using a classical protocol to remove the remaining steric clashes. The system was embedded into a TIP3P (transferable intermolecular potential with 3 points) water box in minimum salt (9.2, 7.3, and 8.2 $\mathrm{nm}, 16,899$ water molecules, $5 \mathrm{~K}^{+}$for a neutral system). ${ }^{62}$ The whole hydrated system was successively minimized, simulated for a short 500 ps trajectory at a constant volume and temperature $(310 \mathrm{~K})$, and further simulated during $200 \mathrm{ps}$ under constant pressure and temperature $(310 \mathrm{~K})$. The Vrescale thermostat ${ }^{63}$ was, used and all the protein heavy atoms were fixed during these two steps. The resulting system was used as the starting point for $1 \mu$ s production runs.
The simulations were performed using Gromacs software, ${ }^{64}$ with the amber99SB-ildn*-q force field ${ }^{65-67}$ including modified parameters for copper ions. ${ }^{68}$ Simulations were performed at a constant temperature $(310 \mathrm{~K})$ with V-rescale thermostat ${ }^{63}$ and pressure ( 1 bar) using a Parrinello-Rahman coupling algorithm. ${ }^{69}$ The integration time step was $2 \mathrm{fs}$, and all bonds were constrained using P-LINCS. ${ }^{70}$ Water molecules were kept rigid using the SETTLE algorithm. ${ }^{71}$ LennardJones interactions were cut off at $1.0 \mathrm{~nm}$. Long-range electrostatic interactions were treated using the Particle Mesh Ewald approach ${ }^{72}$ with a $1.0 \mathrm{~nm}$ direct space cut-off. The neighbor list was updated every $10 \mathrm{ps,} \mathrm{and} \mathrm{the} \mathrm{center-of-}$ mass motion was removed at every step. To keep the copper ion correctly coordinated, distance restraints were applied between $\mathrm{H} 292$, H294, and $\mathrm{H} 296$ coordinating the copper ion, K320 and Y355 forming the LTQ and the copper ion (3.5 with the $\mathrm{N}^{\varepsilon}$ of $\mathrm{H} 292$ and $\mathrm{H} 294$ residues and $\mathrm{N}^{\delta}$ for the $\mathrm{H} 296$ residue and $5 \AA$ with the oxygen atom of LTQ). A calcium ion was added in the LOX model because the crystal structure of the LOXL2 template contains a calcium ion and the six amino acid residues coordinating the calcium ion in LOXL2 (D549, L550, E722, D724, N727, and N728) ${ }^{38}$ are all conserved in the corresponding LOX sequence (D214, L215, E388, D390, N393, and N394). Subsequently, $1 \mu$ s production runs were carried out. The essential dynamics was realized on the last 600 ns of simulation and only on C $\alpha$ atoms of LOX (P213-Y417). All structures were visualized using Visual Molecular Dynamics (VMD, http://www.ks.uiuc.edu/Research/vmd/). ${ }^{73}$

\section{ASSOCIATED CONTENT}

\section{Supporting Information}

The Supporting Information is available free of charge on the ACS Publications website at DOI: 10.1021/acsomega.9b00317.

Alignment of the sequences of human LOX and LOXL2 catalytic domains; superimposition of the 3D structure of the cytokine receptor-like domain of the LOX model generated in this study with the $3 \mathrm{D}$ structure of the CRL domain of the human erythropoietin receptor; $\mathrm{C} \alpha$ RMSD of human LOX during the last $600 \mathrm{~ns}$ using the $400 \mathrm{~ns}$ snapshot as a reference; distribution of the distance between the S232-H259 and Q272-F301 loops, located at the entrance of LOX catalytic site, as a function of the C $\alpha$-RMSD over the last $600 \mathrm{~ns}$ of the simulation using the $400 \mathrm{~ns}$ snapshot as the initial reference; variation of the secondary structure of each amino acid residue of human lysyl oxidase during the 1 $\mu$ s simulation; conserved amino acid residues of LOX involved in cations coordination; cumulative contribution of principal component in the global motion of human lysyl oxidase over the last $600 \mathrm{~ns}$ of simulation; superimposition of the dynamic model of LOX with the LOX model built with Swiss-Model and the crystal structure of LOXL2; geometries of the coordination sites of the cations in the LOX model; analysis of the secondary structure content of our dynamic model of lysyl oxidase, of the model of LOX generated with SwissModel, and of the corresponding domain of the crystal structure of LOXL2 with DSSP; prediction of the effect of LOX mutations on the stability of the model generated in this study; distances measured in the dynamic model of lysyl oxidase between the mutated 
amino acid residues and the copper ion, the calcium ion, and the nitrogen atom $\mathrm{N}^{\varepsilon}$ of LTQ; and coordination numbers of the cations in the LOX model (PDF)

Coordinates of the dynamic model of human LOX generated in this study (PDB)

\section{AUTHOR INFORMATION}

\section{Corresponding Author}

*E-mail: sylvie.ricard-blum@univ-lyon1.fr. Phone: +33(0)4 72 448232.

\section{ORCID $\odot$}

Sylvie Ricard-Blum: 0000-0001-9263-1851

\section{Author Contributions}

"S.D.V. and M.G. contributed equally to this work. S.D.V. and N.B. analyzed the LOX sequence and 3D model, S.D.V. and M.G. performed the molecular modeling, and M.G. ran the molecular dynamics simulations. M.D. and S.R.B. designed the study, participated in data interpretation, drafted the Introduction and Discussion of the manuscript, and revised the manuscript. All authors contributed to the manuscript writing and have given approval to the final version of the manuscript.

\section{Funding}

This work was supported by a grant from the Fondation pour la Recherche Médicale (grant no. DBI20141231336 to S.R.B.), Ligue Nationale contre le Cancer (Comité du Rhône 2017-01CI: UCBL to S.R.-B.), and CNRS (AAP Inter-Instituts 2017-Projet LoxIMoRe to M.D. and S.R.-B.).

\section{Notes}

The authors declare no competing financial interest.

\section{ACKNOWLEDGMENTS}

M.D., N.B., and M.G. are indebted to the research chair MAgICS. Computer hours for MD simulations were kindly performed on the supercenter ROMEO (Reims, France).

\section{ABBREVIATIONS}

AMBER: assisted model building and energy refinement; BMP-1: bone morphogenetic protein-1; CRL: cytokine receptor-like; GROMACS: groningen machine for chemical simulation; LOX: lysyl oxidase; LOXL: lysyl oxidase-like; LTQ: lysine tyrosylquinone; MD: molecular dynamics; PC: principal component; PPLO: Pichia pastoris lysyl oxidase

\section{REFERENCES}

(1) Mäki, J. M. Lysyl Oxidases in Mammalian Development and Certain Pathological Conditions. Histol. Histopathol. 2009, 24, 651660.

(2) Trackman, P. C. Enzymatic and Non-Enzymatic Functions of the Lysyl Oxidase Family in Bone. Matrix Biol. 2016, 52-54, 7-18.

(3) Uzel, M. I.; Scott, I. C.; Babakhanlou-Chase, H.; Palamakumbura, A. H.; Pappano, W. N.; Hong, H. H.; Greenspan, D. S.; Trackman, P. C. Multiple Bone Morphogenetic Protein 1Related Mammalian Metalloproteinases Process pro-Lysyl Oxidase at the Correct Physiological Site and Control Lysyl Oxidase Activation in Mouse Embryo Fibroblast Cultures. J. Biol. Chem. 2001, 276, 22537-22543.

(4) Vallet, S. D.; Miele, A. E.; Uciechowska-Kaczmarzyk, U.; Liwo, A.; Duclos, B.; Samsonov, S. A.; Ricard-Blum, S. Insights into the Structure and Dynamics of Lysyl Oxidase Propeptide, a Flexible Protein with Numerous Partners. Sci. Rep. 2018, 8, 11768.
(5) Grimsby, J. L.; Lucero, H. A.; Trackman, P. C.; Ravid, K.; Kagan, H. M. Role of Lysyl Oxidase Propeptide in Secretion and Enzyme Activity. J. Cell. Biochem. 2010, 111, 1231-1243.

(6) Trackman, P. C. Functional Importance of Lysyl Oxidase Family Propeptide Regions. J. Cell. Commun. Signal. 2018, 45.

(7) Ricard-Blum, S.; Vallet, S. D. Proteases Decode the Extracellular Matrix Cryptome. Biochim. 2016, 122, 300-313.

(8) Ricard-Blum, S.; Vallet, S. D. Fragments Generated upon Extracellular Matrix Remodeling: Biological Regulators and Potential Drugs. Matrix Biol. 2019, 75-76, 170-189.

(9) Chen, X.; Greenaway, F. T. Identification of the Disulfide Bonds of Lysyl Oxidase. J. Neural Transm. 2011, 118, 1111-1114.

(10) Wang, S. X.; Mure, M.; Medzihradszky, K. F.; Burlingame, A. L.; Brown, D. E.; Dooley, D. M.; Smith, A. J.; Kagan, H. M.; Klinman, J. P. A Crosslinked Cofactor in Lysyl Oxidase: Redox Function for Amino Acid Side Chains. Science 1996, 273, 1078-1084.

(11) Wang, S. X.; Nakamura, N.; Mure, M.; Klinman, J. P.; SandersLoehr, J. Characterization of the Native Lysine Tyrosylquinone Cofactor in Lysyl Oxidase by Raman Spectroscopy. J. Biol. Chem. 1997, 272, 28841-28844.

(12) Bollinger, J. A.; Brown, D. E.; Dooley, D. M. The Formation of Lysine Tyrosylquinone (LTQ) Is a Self-Processing Reaction. Expression and Characterization of a Drosophila Lysyl Oxidase. Biochemistry 2005, 44, 11708-11714.

(13) Borel, A.; Eichenberger, D.; Farjanel, J.; Kessler, E.; Gleyzal, C.; Hulmes, D. J. S.; Sommer, P.; Font, B. Lysyl Oxidase-like Protein from Bovine Aorta. Isolation and Maturation to an Active Form by Bone Morphogenetic Protein-1. J. Biol. Chem. 2001, 276, 4894448949.

(14) Li, W.; Nellaiappan, K.; Strassmaier, T.; Graham, L.; Thomas, K. M.; Kagan, H. M. Localization and Activity of Lysyl Oxidase within Nuclei of Fibrogenic Cells. Proc. Natl. Acad. Sci. U. S. A. 1997, 94, 12817-12822.

(15) Giampuzzi, M.; Oleggini, R.; Di Donato, A. Demonstration of in Vitro Interaction between Tumor Suppressor Lysyl Oxidase and Histones $\mathrm{H} 1$ and H2: Definition of the Regions Involved. Biochim. Biophys. Acta 2003, 1647, 245-251.

(16) Li, W.; Nugent, M. A.; Zhao, Y.; Chau, A. N.; Li, S. J.; Chou, I.N.; Liu, G.; Kagan, H. M. Lysyl Oxidase Oxidizes Basic Fibroblast Growth Factor and Inactivates Its Mitogenic Potential. J. Cell. Biochem. 2003, 88, 152-164.

(17) Lucero, H. A.; Ravid, K.; Grimsby, J. L.; Rich, C. B.; DiCamillo, S. J.; Mäki, J. M.; Myllyharju, J.; Kagan, H. M. Lysyl Oxidase Oxidizes Cell Membrane Proteins and Enhances the Chemotactic Response of Vascular Smooth Muscle Cells. J. Biol. Chem. 2008, 283, 2410324117.

(18) Mäki, J. M.; Sormunen, R.; Lippo, S.; Kaarteenaho-Wiik, R.; Soininen, R.; Myllyharju, J. Lysyl Oxidase Is Essential for Normal Development and Function of the Respiratory System and for the Integrity of Elastic and Collagen Fibers in Various Tissues. Am. J. Pathol. 2005, 167, 927-936.

(19) Guo, D.; Regalado, E. S.; Gong, L.; Duan, X.; Santos-Cortez, R. L. P.; Arnaud, P.; Ren, Z.; Cai, B.; Hostetler, E. M.; Moran, R.; et al. LOX Mutations Predispose to Thoracic Aortic Aneurysms and Dissections. Circ. Res. 2016, 118, 928-934.

(20) Lee, V. S.; Halabi, C. M.; Hoffman, E. P.; Carmichael, N.; Leshchiner, I.; Lian, C. G.; Bierhals, A. J.; Vuzman, D.; Mecham, R. P.; Frank, N. Y.; Stitziel, N. O. Loss of Function Mutation inLOXcauses Thoracic Aortic Aneurysm and Dissection in Humans. Proc. Natl. Acad. Sci. U. S. A. 2016, 113, 8759-8764.

(21) Erler, J. T.; Bennewith, K. L.; Nicolau, M.; Dornhöfer, N.; Kong, C.; Le, Q.-T.; Chi, J.-T. A.; Jeffrey, S. S.; Giaccia, A. J. Lysyl Oxidase Is Essential for Hypoxia-Induced Metastasis. Nature 2006, $440,1222-1226$

(22) Cox, T. R.; Rumney, R. M. H.; Schoof, E. M.; Perryman, L.; Høye, A. M.; Agrawal, A.; Bird, D.; Latif, N. A.; Forrest, H.; Evans, H. $\mathrm{R}$; et al. The Hypoxic Cancer Secretome Induces Pre-Metastatic Bone Lesions through Lysyl Oxidase. Nature 2015, 522, 106-110. 
(23) Cox, T. R.; Gartland, A.; Erler, J. T. Lysyl Oxidase, a Targetable Secreted Molecule Involved in Cancer Metastasis. Cancer Res. 2016, 76, 188-192.

(24) Gartland, A.; Erler, J. T.; Cox, T. R. The Role of Lysyl Oxidase, the Extracellular Matrix and the Pre-Metastatic Niche in Bone Metastasis. J. Bone Oncol. 2016, 5, 100-103.

(25) Pastel, E.; Price, E.; Sjöholm, K.; McCulloch, L. J.; Rittig, N.; Liversedge, N.; Knight, B.; Møller, N.; Svensson, P.-A.; Kos, K. Lysyl Oxidase and Adipose Tissue Dysfunction. Metab., Clin. Exp. 2018, 78, $118-127$.

(26) Lopez, K. M.; Greenaway, F. T. Identification of the CopperBinding Ligands of Lysyl Oxidase. J. Neural Transm. 2011, 118, $1101-1109$.

(27) Papadantonakis, N.; Matsuura, S.; Ravid, K. Megakaryocyte Pathology and Bone Marrow Fibrosis: The Lysyl Oxidase Connection. Blood 2012, 120, 1774-1781.

(28) Aumiller, V.; Strobel, B.; Romeike, M.; Schuler, M.; Stierstorfer, B. E.; Kreuz, S. Comparative Analysis of Lysyl Oxidase (like) Family Members in Pulmonary Fibrosis. Sci. Rep. 2017, 7, 149.

(29) Barker, H. E.; Cox, T. R.; Erler, J. T. The Rationale for Targeting the LOX Family in Cancer. Nat. Rev. Cancer 2012, 12, $540-552$.

(30) Trackman, P. C. Lysyl Oxidase Isoforms and Potential Therapeutic Opportunities for Fibrosis and Cancer. Expert Opin. Ther. Targets 2016, 20, 935-945.

(31) Harlow, C. R.; Wu, X.; van Deemter, M.; Gardiner, F.; Poland, C.; Green, R.; Sarvi, S.; Brown, P.; Kadler, K. E.; Lu, Y.; et al. Targeting Lysyl Oxidase Reduces Peritoneal Fibrosis. PLoS One 2017, 12, No. e0183013.

(32) Pinnell, S. R.; Martin, G. R. The Cross-Linking of Collagen and Elastin: Enzymatic Conversion of Lysine in Peptide Linkage to AlphaAminoadipic-Delta-Semialdehyde (Allysine) by an Extract from Bone. Proc. Natl. Acad. Sci. U. S. A. 1968, 61, 708-716.

(33) Duff, A. P.; Cohen, A. E.; Ellis, P. J.; Kuchar, J. A.; Langley, D. B.; Shepard, E. M.; Dooley, D. M.; Freeman, H. C.; Guss, J. M. The Crystal Structure of Pichia Pastoris Lysyl Oxidase. Biochemistry 2003, 42, 15148-15157.

(34) Duff, A. P.; Cohen, A. E.; Ellis, P. J.; Hilmer, K.; Langley, D. B.; Dooley, D. M.; Freeman, H. C.; Guss, J. M. The $1.23 \AA$ Structure Pichia Pastoris Lysyl Oxidase Reveals a Lysine-Lysine Cross-Link. Acta Crystallogr. D Biol. Crystallogr. 2006, 62, 1073-1084.

(35) Kagan, H. M.; Ryvkin, F. Lysyl Oxidase and Lysyl Oxidase-Like Enzymes. In The Extracellular Matrix: an Overview; Biology of Extracellular Matrix; Mecham, R. P., Ed.; Springer: Berlin Heidelberg, 2011; pp 303-335, DOI: 10.1007/978-3-642-16555-9 9.

(36) Bhuvanasundar, R.; John, A.; Sulochana, K. N.; Coral, K.; Deepa, P. R.; Umashankar, V. A Molecular Model of Human Lysyl Oxidase (LOX) with Optimal Copper Orientation in the Catalytic Cavity for Induced Fit Docking Studies with Potential Modulators. Bioinformation 2014, 10, 406-412.

(37) Krebs, C. J.; Krawetz, S. A. Lysyl Oxidase Copper-Talon Complex: A Model. Biochim. Biophys. Acta 1993, 1202, 7-12.

(38) Zhang, X.; Wang, Q.; Wu, J.; Wang, J.; Shi, Y.; Liu, M. Crystal Structure of Human Lysyl Oxidase-like 2 (HLOXL2) in a Precursor State. Proc. Natl. Acad. Sci. U. S. A. 2018, 115, 3828-3833.

(39) Song, Y.; DiMaio, F.; Wang, R. Y.-R.; Kim, D.; Miles, C.; Brunette, T.; Thompson, J.; Baker, D. High-Resolution Comparative Modeling with RosettaCM. Structure 2013, 21, 1735-1742.

(40) Kim, Y.; Boyd, C. D.; Csiszar, K. A New Gene with Sequence and Structural Similarity to the Gene Encoding Human Lysyl Oxidase. J. Biol. Chem. 1995, 270, 7176-7182.

(41) Livnah, O.; Stura, E. A.; Middleton, S. A.; Johnson, D. L.; Jolliffe, L. K.; Wilson, I. A. Crystallographic Evidence for Preformed Dimers of Erythropoietin Receptor before Ligand Activation. Science 1999, 283, 987-990.

(42) Unni, S.; Huang, Y.; Hanson, R. M.; Tobias, M.; Krishnan, S.; Li, W. W.; Nielsen, J. E.; Baker, N. A. Web Servers and Services for Electrostatics Calculations with APBS and PDB2PQR. J. Comput. Chem. 2011, 32, 1488-1491.
(43) Oldfield, R. N.; Johnston, K. A.; Limones, J.; Ghilarducci, C.; Lopez, K. M. Identification of Histidine 303 as the Catalytic Base of Lysyl Oxidase via Site-Directed Mutagenesis. Protein J. 2018, 37, 4757.

(44) Gacheru, S. N.; Trackman, P. C.; Shah, M. A.; O’Gara, C. Y.; Spacciapoli, P.; Greenaway, F. T.; Kagan, H. M. Structural and Catalytic Properties of Copper in Lysyl Oxidase. J. Biol. Chem. 1990, 265, 19022-19027.

(45) Suzek, B. E.; Wang, Y.; Huang, H.; McGarvey, P. B.; Wu, C. H. UniRef Clusters: A Comprehensive and Scalable Alternative for Improving Sequence Similarity Searches. Bioinformatics 2015, 31, 926-932.

(46) Smart, O. S.; Neduvelil, J. G.; Wang, X.; Wallace, B. A.; Sansom, M. S. HOLE: A Program for the Analysis of the Pore Dimensions of Ion Channel Structural Models. J. Mol. Graph. 1996, $14,354-360$.

(47) Wilson, J. J.; Matsushita, O.; Okabe, A.; Sakon, J. A Bacterial Collagen-Binding Domain with Novel Calcium-Binding Motif Controls Domain Orientation. EMBO J. 2003, 22, 1743-1752.

(48) Laimer, J.; Hofer, H.; Fritz, M.; Wegenkittl, S.; Lackner, P. MAESTRO-Multi Agent Stability Prediction upon Point Mutations. BMC Bioinf. 2015, 16, 116.

(49) Laimer, J.; Hiebl-Flach, J.; Lengauer, D.; Lackner, P. MAESTROweb: A Web Server for Structure-Based Protein Stability Prediction. Bioinformatics 2016, 32, 1414-1416.

(50) Waterhouse, A.; Bertoni, M.; Bienert, S.; Studer, G.; Tauriello, G.; Gumienny, R.; Heer, F. T.; de Beer, T. A. P.; Rempfer, C.; Bordoli, L.; et al. SWISS-MODEL: Homology Modelling of Protein Structures and Complexes. Nucleic Acids Res. 2018, 46, W296-W303.

(51) Lenffer, J.; Lai, P.; El Mejaber, W.; Khan, A. M.; Koh, J. L. Y.; Tan, P. T. J.; Seah, S. H.; Brusic, V. CysView: Protein Classification Based on Cysteine Pairing Patterns. Nucleic Acids Res. 2004, 32, W350-355.

(52) Montgomerie, S.; Cruz, J. A.; Shrivastava, S.; Arndt, D.; Berjanskii, M.; Wishart, D. S. PROTEUS2: A Web Server for Comprehensive Protein Structure Prediction and Structure-Based Annotation. Nucleic Acids Res. 2008, 36, W202-209.

(53) Ishida, T.; Kinoshita, K. Prediction of Disordered Regions in Proteins Based on the Meta Approach. Bioinformatics 2008, 24, 1344-1348.

(54) Dosztányi, Z.; Mészáros, B.; Simon, I. ANCHOR: Web Server for Predicting Protein Binding Regions in Disordered Proteins. Bioinformatics 2009, 25, 2745-2746.

(55) Mészáros, B.; Simon, I.; Dosztányi, Z. Prediction of Protein Binding Regions in Disordered Proteins. PLoS Comput. Biol. 2009, 5, No. e1000376.

(56) Malhis, N.; Jacobson, M.; Gsponer, J. MoRFchibi SYSTEM: Software Tools for the Identification of MoRFs in Protein Sequences. Nucleic Acids Res. 2016, 44, W488-493.

(57) Kim, D. E.; Chivian, D.; Baker, D. Protein Structure Prediction and Analysis Using the Robetta Server. Nucleic Acids Res. 2004, 32, W526-531.

(58) Söding, J.; Biegert, A.; Lupas, A. N. The HHpred Interactive Server for Protein Homology Detection and Structure Prediction. Nucleic Acids Res. 2005, 33, W244-248.

(59) Alva, V.; Nam, S.-Z.; Söding, J.; Lupas, A. N. The MPI Bioinformatics Toolkit as an Integrative Platform for Advanced Protein Sequence and Structure Analysis. Nucleic Acids Res. 2016, 44, W410-415.

(60) Moore, R. H.; Spies, M. A.; Culpepper, M. B.; Murakawa, T.; Hirota, S.; Okajima, T.; Tanizawa, K.; Mure, M. Trapping of a Dopaquinone Intermediate in the TPQ Cofactor Biogenesis in a Copper-Containing Amine Oxidase from Arthrobacter Globiformis. J. Am. Chem. Soc. 2007, 129, 11524-11534.

(61) Ray, A.; Lindahl, E.; Wallner, B. Improved Model Quality Assessment Using ProQ2. BMC Bioinf. 2012, 13, 224.

(62) Jorgensen, W. L.; Chandrasekhar, J.; Madura, J. D.; Impey, R. W.; Klein, M. L. Comparison of Simple Potential Functions for Simulating Liquid Water. J. Chem. Phys. 1983, 79, 926-935. 
(63) Bussi, G.; Donadio, D.; Parrinello, M. Canonical Sampling through Velocity Rescaling. J. Chem. Phys. 2007, 126, No. 014101.

(64) Pronk, S.; Páll, S.; Schulz, R.; Larsson, P.; Bjelkmar, P.; Apostolov, R.; Shirts, M. R.; Smith, J. C.; Kasson, P. M.; van der Spoel, D.; et al. GROMACS 4.5: A High-Throughput and Highly Parallel Open Source Molecular Simulation Toolkit. Bioinformatics 2013, 29, 845-854.

(65) Lindorff-Larsen, K.; Piana, S.; Palmo, K.; Maragakis, P.; Klepeis, J. L.; Dror, R. O.; Shaw, D. E. Improved Side-Chain Torsion Potentials for the Amber Ff99SB Protein Force Field. Proteins 2010, 78, 1950-1958.

(66) Best, R. B.; Hummer, G. Optimized Molecular Dynamics Force Fields Applied to the Helix-Coil Transition of Polypeptides. J. Phys. Chem. B 2009, 113, 9004-9015.

(67) Best, R. B.; Mittal, J.; Feig, M.; MacKerell, A. D., Jr. Inclusion of Many-Body Effects in the Additive CHARMM Protein CMAP Potential Results in Enhanced Cooperativity of $\alpha$-Helix and $\beta$-Hairpin Formation. Biophys. J. 2012, 103, 1045-1051.

(68) Torras, J.; Alemán, C. Determination of New $\mathrm{Cu}^{+}, \mathrm{Cu}^{2+}$, and $\mathrm{Zn}^{2+}$ Lennard-Jones Ion Parameters in Acetonitrile. J. Phys. Chem. B 2013, 117, 10513-10522.

(69) Parrinello, M.; Rahman, A. Polymorphic Transitions in Single Crystals: A New Molecular Dynamics Method. J. Appl. Phys. 1981, 52, $7182-7190$

(70) Hess, B. P-LINCS: A Parallel Linear Constraint Solver for Molecular Simulation. J. Chem. Theory Comput. 2008, 4, 116-122.

(71) Miyamoto, S.; Kollman, P. A. Settle: An Analytical Version of the SHAKE and RATTLE Algorithm for Rigid Water Models. J. Comput. Chem. 1992, 13, 952-962.

(72) Cheatham, T. E. I.; Miller, J. L.; Fox, T.; Darden, T. A.; Kollman, P. A. Molecular Dynamics Simulations on Solvated Biomolecular Systems: The Particle Mesh Ewald Method Leads to Stable Trajectories of DNA, RNA, and Proteins. J. Am. Chem. Soc. 1995, 117, 4193-4194.

(73) Humphrey, W.; Dalke, A.; Schulten, K. VMD: Visual Molecular Dynamics. J. Mol. Graph. 1996, 14, 33-38. 